\title{
A New Topological Index for Capacity Allocation Problem in Survivable Networks
}

\author{
William Liu ${ }^{1}$, Harsha Sirisena ${ }^{1}$, Krzysztof Pawlikowski $^{2}$ and Andreas Willig ${ }^{2}$ \\ ${ }^{1}$ Electrical and Computer Engineering \\ ${ }^{2}$ Computer Science and Software Engineering \\ University of Canterbury, Christchurch, New Zealand \\ Email: william.liu@elec.canterbury.ac.nz, \{harsha.sirisena, krys.pawlikowski, andreas.willig\}@canterbury.ac.nz
}

\begin{abstract}
In this paper, we propose a new topological index, which is a numerical descriptor that characterizes survivable network topologies. A monotonically decreasing power law relationship can be found between this index and the total capacity allocation in the network. The new topological index is calculated based on the algebraic connectivity, which is adopted from spectral graph theory, more specifically it is based on the second-smallest eigenvalue of the Laplacian matrix of the network topology. Instead of the average nodal degree index that is usually used to characterize network connectivity in studies of the capacity allocation problem, our results suggest that this new topological index more accurately predicts the total capacity and is more informative. It can be used in studies on quantitative structure-performance relationships, in which the network performance or other properties of network are correlated with their topological structure. Extensive case studies confirm that the connections between the total capacity and its network structure can be well described by this topological index in network survivability studies.
\end{abstract}

Keywords- Network survivability; capacity allocation; network connectivity; algebraic connectivity metric; second-smallest eigenvalue; Laplacian matrix; Shared Backup Path Protection; Integer Linear Programming.

\section{INTRODUCTION}

Survivability is one of the most important design issues of future multi-service Next Generation Networks (NGNs). Designing survivable networks involves pre-planning the network topology jointly with the allocation of spare link capacities (Spare Capacity Allocation or SCA problem) to be used in case of failures [1]. The performance of survivable routing protocols, the robustness of the network under failures and traffic engineering depend crucially on the topology of a given network. Network robustness can be characterized by the network topological connectivity, which determines the level of immunity / survivability of the network if failures of nodes and links occur. In general, as the network connectivity increases, one can find more node- and link-disjoint paths between pairs of communicating nodes, the pre-determined working and spare/protection paths can become shorter, and the additionally available paths can be used to reduce the requirements on the spare capacity. Thus the capacity needed for working and protection capacities decreases. In addition, the more disjoint protection paths can be distinguished, the higher the spare capacity sharing that can be obtained in the shared backup path protection (SBPP) survivable routing scheme $[1,2]$. These dependencies underlie the search for an optimum and cost-efficient topology in network survivability design.

The SCA design of survivable networks of given topologies has been subject to much research in recent years. Most studies [3-7] use the average nodal degree index for quantifying the relationship between capacity allocation and network connectivity. For a given network topology with bidirectional links, the average nodal degree $\bar{d}$ is defined as the ratio of twice the number of links to the number of nodes. The simulation studies presented in [3-7] have concentrated on showing how the working and spare capacity requirements of networks with various topologies vary with the average nodal degree index. Despite of the wide adoption of the average nodal degree index in these studies, we argue that this index is only a coarse indicator of how sparse or dense a given topology is. It carries insufficient information about the networks topological structure. Furthermore, employing the average nodal degree for describing the network's characteristics may lead to misleading findings e.g., several networks have the same average node degree and thus the same network connectivity characteristic, their total capacity allocated should be similar, but they can be totally different [8]. We suggest a more informative and accurate topological index: the ratio of the algebraic connectivity $\lambda_{2}(G)$ to the network mean distance $\rho(G)$, i.e., $\lambda_{2}(G) / \rho(G)$, here the network mean distance measures the hop count. The algebraic connectivity is defined as the second-smallest eigenvalue of the Laplacian matrix of a given topology, and it is an accurate descriptor of network characteristics such as connectivity in a broader spectrum of graphs [9]. It is one of the key invariants of a graph and has found a wide range of applications. One of its desirable properties we have used is that its magnitude (if non-zero) is related with the number of node- and link-disjoint paths, it is thus an important design input for the total capacity allocation in survivable network design.

The structure of this paper is outlined as follows. Section II introduces the algebraic connectivity together with its theoretical ramification, and then the new proposed topological index is defined. In Section III we introduce the Integer Linear Programming (ILP) model of the shared backup path protection (SBPP) scheme, which is used to evaluate the correlation between the new topological index and capacity allocation. The results of our extensive case studies conducted for experimental comparison of the properties of different topological indices are reported in Section IV. Final conclusions are drawn in Section V. 


\section{TOPOlOGiCAL MEASURES OF NETWORK ROBUSTNESS}

In general, topological measures are functions of the network topology $G(N, L)$. The number of nodes $N$ and the number of links $L$ are the mostly regarded parameters of a network, but they are not its metrics. Many measures depend on the size of the network $N$ and the number of links $L$. For example, the degree $d_{i}$ of a node $i$ in a network is defined as the number of links that are incident on node $i$. The node degree represents an important characteristic of a node, as a node with larger $d_{i}$ can usually serve more streams of data. To simplify the following exposition, we regard $D$ as a generic random variable giving the node degree (ranging from 0 to the maximum degree $d_{\max }$ ) of a given node in a network. The node degree distribution of this network then corresponds to the probability mass function of the random variable $D$ for this network, i.e. it can be defined as the set of $\operatorname{Pr}[D=k]$, for $1 \leq k \leq d_{\max }$, where $\operatorname{Pr}[D=k]$ represents the fraction of nodes in the network with node degree $k$. In other words, it is the probability that a randomly chosen node has degree $k$. The average nodal degree, denoted as $\bar{d}$, is defined as the expectation of $D$, for which in a network with $N$ nodes $L$ links we have:

$$
\bar{d}=E[D]=\sum_{k=1}^{d_{\max }} k \cdot \operatorname{Pr}[D=k]=\frac{2 L}{N}
$$

In addition, the node connectivity $v(G)$ and the link connectivity $e(G)$ of a network are defined as the minimal number of nodes with all links that are incident to them and links, respectively, which have to be removed in order to disconnect that network. They appear to be natural quantifiers for network robustness, but are difficult to compute for large networks. Among the structural metrics mentioned above, the average nodal degree $\bar{d}$ is widely used for quantifying the relationship between the network connectivity and the amount of allocated capacity, see for example $[5,6]$. The simulation results presented there show how the total amount of working and spare capacity allocated in different network topologies, vary according to their different average nodal degrees. Despite of this wide adoption of the average nodal degree in survivability studies, we argue that it is only a coarse indicator of how sparse or dense a given topology is and it cannot differentiate the shape and size of the different topologies. Therefore, we suggest using a more informative topological index, which is based on the algebraic connectivity adopted from spectral graph theory. We will provide numerical evidence for this statement in Section IV.

One of the main goals of the spectral graph theory is to deduce the principal properties and the structure of a graph from its spectrum [9]. Graph spectral analysis can be used to reveal the fundamental properties of a graph through geometric, analytic and algebraic techniques.

Let $G(N, L)$ be a network with $N$ nodes from set $\mathcal{N}$ and $L$ links from set $\mathcal{L}$, i.e., $N=|\mathcal{N}|$ and $L=|\mathcal{L}|$. The network $G$ can be represented by its adjacency matrix, $\boldsymbol{A}(G)$, which is the $N \times N$ matrix whose $(i, j)$-th entry is " 1 " if node $i$ is connected to node $j$, i.e., link $(i, j) \in \mathcal{L}$, or 0 otherwise. The diagonal entries of $\boldsymbol{A}(G)$ are defined to be 0 . Let $\boldsymbol{D}(G)$ be the $N x N$ diagonal matrix with entries $\boldsymbol{D}_{i, i}=d_{i}$, where $d_{i}$ is the degree of the $i$-th node of $G$. The Laplacian matrix, $\boldsymbol{Q}(G)$, of the network $G$ is defined as:

$$
\boldsymbol{Q}(G)=\boldsymbol{D}(G)-\boldsymbol{A}(G)
$$

For the Laplacian matrix $\boldsymbol{Q}(G)$, an $N$-dimensional and non-zero vector $\overrightarrow{\boldsymbol{x}}$ is an eigenvector if there is a scalar $\lambda$, such that $\boldsymbol{Q} \overrightarrow{\boldsymbol{x}}=\lambda \overrightarrow{\boldsymbol{x}}$. Here the $\lambda$ is the eigenvalue of $\boldsymbol{Q}(G)$ corresponding to the eigenvector $\overrightarrow{\boldsymbol{x}}$. By its definition, $\boldsymbol{Q}(G)$ is a real symmetric and positive semi-definite matrix, thus all of its $N$ eigenvalues are real and non-negative. Notice that the allones vector is an eigenvector of any Laplacian matrix $\boldsymbol{Q}$ and its associated eigenvalue is 0 . A Fiedler vector $\overrightarrow{\boldsymbol{x}}=\left(x_{1}, \ldots, x_{N}\right)$ satisfies:

$$
\sum_{i=1}^{N} x_{i}=0
$$

since the all-ones vector is an eigenvector of the Laplacian matrix $\boldsymbol{Q}(G)$ and the eigenvectors of a symmetric matrix are orthogonal. The Laplacian matrix $\boldsymbol{Q}(G)$ has $N$ nonnegative real eigenvalues:

$$
0=\lambda_{1} \leq \lambda_{2} \leq \cdots \lambda_{N}
$$

It can be seen that 0 is always an eigenvalue of $\boldsymbol{Q}(G)$, and that $\overrightarrow{\mathbf{1}}=(1,1, \ldots 1)^{T}$ is the corresponding eigenvector. The set of eigenvalues of the Laplacian matrix $\boldsymbol{Q}(G)$ for a given graph is called the Laplacian spectrum of $G$. These eigenvalues are closely related to almost all major invariants of a graph [9]. In particular, an important role is played by the second-smallest eigenvalue $\lambda_{2}(G)$, known as the algebraic connectivity, or Fiedler value in $[11,12]$. It has been shown that the algebraic connectivity is only equal to zero if $G$ is disconnected. In general, the number of eigenvalues of the Laplacian matrix $\boldsymbol{Q}(G)$ that have value zero coincides with the number of disconnected components of $G$. Moreover, the larger the algebraic connectivity of a network is, the more difficult it is to break up the network into separate components. Thus, the algebraic connectivity is a commonly accepted metric for studying network properties as their connectivity and minimum cut-sets. In addition, the algebraic connectivity is also widely studied in various fields of mathematics, for example in combinatorial optimization [13].

We now consider the practical calculation of the algebraic connectivity. Since $\boldsymbol{Q}(G)$ is a symmetric matrix, the Rayleigh quotient of $\overrightarrow{\boldsymbol{x}}$ with respect to $\boldsymbol{Q}(G)$ is:

$$
\frac{\vec{x}^{T} Q(G) \vec{x}}{\vec{x}^{T} \vec{x}}
$$

The algebraic connectivity $\lambda_{2}$ of the network $G$ satisfies:

$$
\lambda_{2}=\min _{\vec{x} \perp(1,1, \ldots, 1)} \frac{\overrightarrow{\boldsymbol{x}}^{T} \boldsymbol{Q}(G) \overrightarrow{\boldsymbol{x}}}{\overrightarrow{\boldsymbol{x}}^{T} \overrightarrow{\boldsymbol{x}}}
$$


In equation (6), the minimum value of $\lambda_{2}$ occurs only when $\overrightarrow{\boldsymbol{x}}$ is the Fiedler vector. For any vector $\overrightarrow{\boldsymbol{x}} \in R^{N}$, we have:

$$
\overrightarrow{\boldsymbol{x}}^{T} \boldsymbol{Q}(G) \overrightarrow{\boldsymbol{x}}=\sum_{(i, j) \in \mathcal{L}}\left(x_{i}-x_{j}\right)^{2}
$$

We denote the standard norm of a vector $\overrightarrow{\boldsymbol{x}}$ in Euclidean space by $\|\overrightarrow{\boldsymbol{x}}\|=\sqrt{\boldsymbol{x}^{T} \boldsymbol{x}}$ and the algebraic connectivity metric $\lambda_{2}$ can be calculated from the following lemma [14].

Lemma Let $G(N, L)$ be a given network. Then $\lambda_{2}$, the algebraic connectivity of $G$, is given by:

$$
\lambda_{2}=\min \frac{\sum_{(i, j) \in \mathcal{L}}\left\|\overrightarrow{x_{i}}-\overrightarrow{x_{j}}\right\|^{2}}{\sum_{i=1}^{N}\left\|\overrightarrow{x_{i}}\right\|^{2}}
$$

where the minimum is taken over the vectors $\left\{\overrightarrow{x_{1}} \ldots \ldots \overrightarrow{x_{N}}\right\} \subset R^{N}$ such that $\sum_{i=1}^{N} \overrightarrow{x_{i}}=\overrightarrow{\mathbf{0}}$, and $\overrightarrow{\mathbf{0}}$ denotes the allzeros vector. The magnitude of the value $\lambda_{2}$ reflects how well connected the network $G$ is. Here, we also recall those two traditional concepts in network connectivity:

- Link Connectivity $e(G)$,which is defined as the minimal number of links whose removal would result in losing connectivity of the network $G$;

- Node Connectivity $v(G)$, which is defined as the minimal number of nodes whose removal together with adjacent links, would result in losing connectivity of network $G$.

The algebraic connectivity $\lambda_{2}$ is upper bounded by these two metrics and is claimed to be a better robustness metric [12]. In an incomplete graph, we have the following inequality:

$$
\lambda_{2}(G) \leq v(G) \leq e(G) \leq d_{\text {min }}(G)
$$

The algebraic connectivity $\lambda_{2}$ is a more accurate measure with respect to the network connectivity than either the node or the link connectivity. Unlike those two traditional connectivity metrics, the algebraic connectivity depends on the number of nodes, as well as the way in which nodes are connected. The node connectivity $v(G)$ is always no smaller than the link connectivity $e(G)$, since deleting one node incident on each link in a cut-set succeeds in disconnecting the network. The minimum node degree the in network, denoted as $d_{\text {min }}(G)$, is an upper bound of both the link and node connectivity, since deleting all its neighbors (i.e., the links to all its neighbors) disconnects the network into one large and one single-node component.

As shown in [10], the algebraic connectivity $\lambda_{2}$ is also closely related to some other graph invariants. One of the most interesting connections is its relation to the network mean distance, $\rho(G)$. The network mean distance here is defined as the average of all hop count between distinct nodes of the graph. In some sense, this metric can measure the size and shape of the graph. In [14], the bounds on the mean distance $\rho(G)$ are derived. Its lower bound is:

$$
(N-1) \rho(G) \geq \frac{2}{\lambda_{2}}+\frac{N-2}{2}
$$

and its upper bound is:

$$
\rho(G) \leq \frac{N}{N-1}\left(\left\lceil\frac{\Delta(G)+\lambda_{2}}{4 \lambda_{2}} \ln (N-1)\right\rceil+\frac{1}{2}\right)
$$

Here $G$ is a given network with $N$ nodes, $\lambda_{2}$ is its algebraic connectivity, and $\Delta(G)$ is the maximal node degree. As our case studies results show, the information provided by the network's mean distance $\rho(G)$ is useful for comparing different network topologies.

On the basis of the above properties of the algebraic connectivity metric, we propose a new topological index: the ratio of the algebraic connectivity $\lambda_{2}(G)$ to network mean distance $\rho(G)$, i.e., $\lambda_{2}(G) / \rho(G)$. We use this as a topological index to reflect network topological properties such as the connectivity, size and shape of a given topology. Since these topological properties have impacts on the total capacity allocation in network survivability design, it is worthwhile to study the correlation between the new topological index and total capacity. On this basis, we formulate the hypothesis that by leveraging the topological index of a given network topology, it can lead to optimization of both working and spare capacity allocations.

\section{SHARED BackuP PATH PRotection Scheme}

In order to evaluate the correlation between the new algebraic connectivity-based topological index versus the average nodal degree and capacity allocation in survivable network design, we use the shared backup path protection (SBPP) spare capacity allocation scheme. The AMPL model for SBPP has been adopted from [15].

Let $\boldsymbol{p} \boldsymbol{F}$ denote the set of links whose failure disrupts working capacity for a given pair $(o, d)$ of nodes. For each $f \in$ $\boldsymbol{p} \boldsymbol{F}, \boldsymbol{p} \boldsymbol{D}_{f}$ denotes the set of $(o, d)$ pairs affected by the failure of link $f$. Since the capacity for a demand pair, $r_{o d}$, can be split among multiple paths, $q_{f(o d)}$ denotes the total amount of capacity from $o$ to $d$ that must be restored when $f$ fails. Let us define the following decision variables:

- $t_{p f}$ : the protection capacity on path $p$ when $\operatorname{link} f$ fails,

- $t_{e}$ : the total protection capacity on link $e$,

- $w_{e}$ : the working capacity,

- $S_{e}$ : the total capacity on link $e$,

- $J_{o, d}$ : the paths that can be used to satisfy demand $(o, d)$,

- $L p_{e}$ : the protection paths that use link $e$.

Using this notation, the shared protection model uses three sets of constraints to determine the values for $S_{e}$. The demand for spare capacity is defined by the constraints:

$$
\sum_{p \in J_{o d} \backslash L p_{f}} t_{p f}=q_{f(o d)} \quad \forall f \in \boldsymbol{p} \boldsymbol{F},(o, d) \in \boldsymbol{p} \boldsymbol{D}_{f}
$$

Note that a protection path containing link $f$ cannot be used to protect against failures of link $f$. Conversion of protection path flows to link spare capacity requirements is accomplished by the following inequalities 


$$
\sum_{p \in L p_{e}} t_{p f} \leq t_{e} \quad \forall e \in \boldsymbol{L}, f \in \boldsymbol{p} \boldsymbol{F}
$$

That is, the spare capacity must be sufficient to accommodate the failure that produces the largest traffic disruption. Provisioning of total traffic on a link is determined by the working capacity on the link plus the spare capacity on the link. From $L$ equations, one gets a solution:

$$
S_{e}=w_{e}+t_{e}, \forall e \in \boldsymbol{L}
$$

The above AMPL model uses concepts of spare capacity sharing and path-based protection derived from SBPP algorithm, see [15] for more details.

\section{CASE STUdies}

In our case studies we have used ten different topologies [16], specified in Figure 1 and Table 1, to investigate the correlation between the topological indices and the total capacity allocated. Without loss of generality, and for easier comparison of results, we have assumed symmetric traffic flows only, i.e., one unit of bandwidth demand between any pair of nodes. The SBPP ILP model is solved using AMPL/CPLEX $11.1[17,18]$.
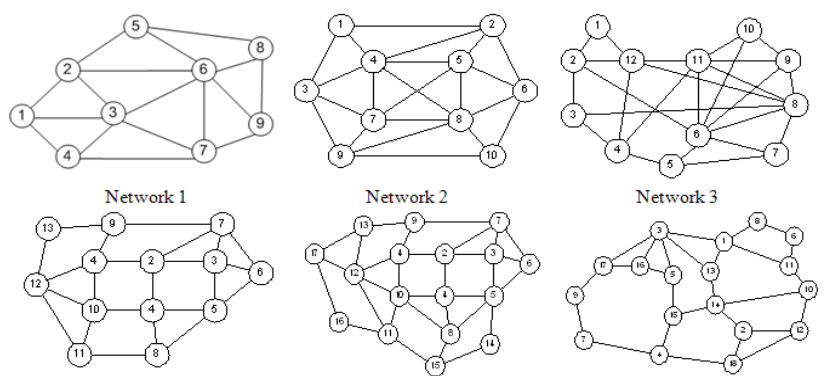

Network 2

Network 3
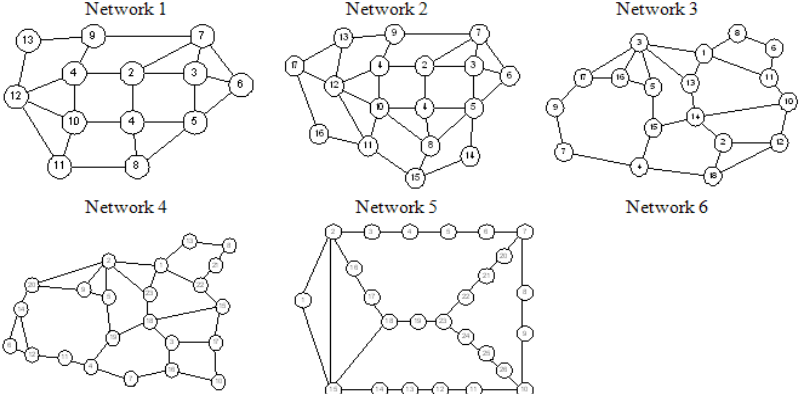

Network 5

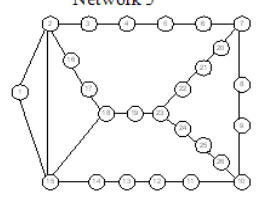

Network 7
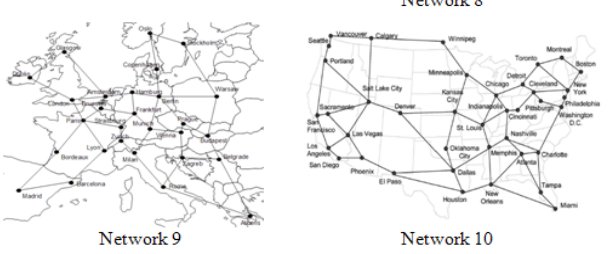

Fig.1 Ten referenced network topologies

Table 1 Network Information for ten referenced networks

\begin{tabular}{|c|c|c|c|}
\hline Network No. & $\boldsymbol{N}$ & $\boldsymbol{L}$ & $\begin{array}{c}\text { Average Node } \\
\text { Degree }\end{array}$ \\
\hline $\mathbf{1}$ & 9 & 17 & 3.7778 \\
\hline $\mathbf{2}$ & 10 & 22 & 4.4000 \\
\hline $\mathbf{3}$ & 12 & 25 & 4.1667 \\
\hline $\mathbf{5}$ & 13 & 23 & 3.5385 \\
\hline $\mathbf{6}$ & 17 & 31 & 3.6471 \\
\hline $\mathbf{7}$ & 18 & 27 & 3.0000 \\
\hline $\mathbf{8}$ & 23 & 33 & 2.8696 \\
\hline $\mathbf{9}$ & 26 & 30 & 2.3077 \\
\hline $\mathbf{1 0}$ & 28 & 41 & 2.9286 \\
\hline
\end{tabular}

In our case studies, we have considered the different topologies, both in terms of their size and shape. We propose to use the new topological index, i.e. $\lambda_{2}(G) / \rho(G)$, to quantify arbitrary topologies. To show that accurate knowledge of the network mean distance $\rho(G)$ is required, we also use its lower bound, $\rho_{\text {lower }}(G)$ and upper bound, $\rho_{\text {upper }}(G)$ estimated by equations (13-14). The exact mean distance $\rho_{\text {exact }}(G)$ can be calculated by the standard graph toolbox in MATLAB [19].

Table 2 Total capacities vs. four different topological indices for ten reference networks

\begin{tabular}{|c|c|c|c|c|c|c|c|}
\hline $\begin{array}{c}\text { Network } \\
\text { No. }\end{array}$ & $\boldsymbol{\Lambda}_{\mathbf{2}}$ & $\boldsymbol{\Delta}$ & $\begin{array}{c}\text { Average Node } \\
\text { Degree }\end{array}$ & $\boldsymbol{\lambda}_{\mathbf{2}} / \boldsymbol{\rho}_{\text {exact }}$ & $\boldsymbol{\lambda}_{\mathbf{2}} / \boldsymbol{\rho}_{\text {lower }}$ & $\boldsymbol{\lambda}_{\mathbf{2}} / \boldsymbol{\rho}_{\text {upper }}$ & $\begin{array}{c}\text { Total } \\
\text { capacity }\end{array}$ \\
\hline 1 & 1.2954 & 6 & 3.7778 & 0.7904 & 2.0547 & 0.3933 & 171 \\
\hline 2 & 1.7985 & 6 & 4.4000 & 1.1399 & 3.1666 & 0.6796 & 193 \\
\hline 3 & 1.2263 & 7 & 4.1667 & 0.7226 & 2.0342 & 0.2795 & 344 \\
\hline 4 & 0.8519 & 4 & 3.5385 & 0.4102 & 1.3026 & 0.2223 & 456 \\
\hline 5 & 0.6339 & 5 & 3.6471 & 0.2694 & 0.9519 & 0.0968 & 892 \\
\hline 6 & 0.4057 & 5 & 3.0000 & 0.1503 & 0.5334 & 0.0406 & 1351 \\
\hline 7 & 0.2375 & 5 & 2.8696 & 0.0722 & 0.2761 & 0.0133 & 2912 \\
\hline 8 & 0.1612 & 4 & 2.3077 & 0.0384 & 0.1651 & 0.0075 & 4642 \\
\hline 9 & 0.1750 & 5 & 2.9286 & 0.0491 & 0.1934 & 0.0069 & 4785 \\
\hline 10 & 0.1107 & 5 & 3.1282 & 0.0263 & 0.1150 & 0.0026 & 10346 \\
\hline
\end{tabular}

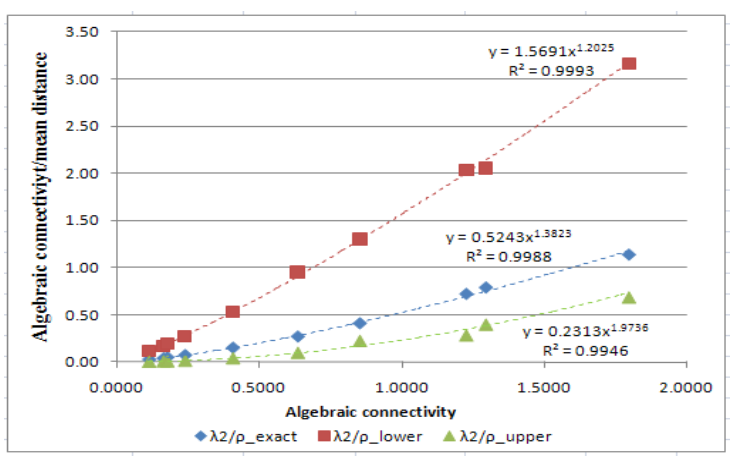

Fig. 2 Correlation between the algebraic connectivity and algebraic connectivity based topological indices

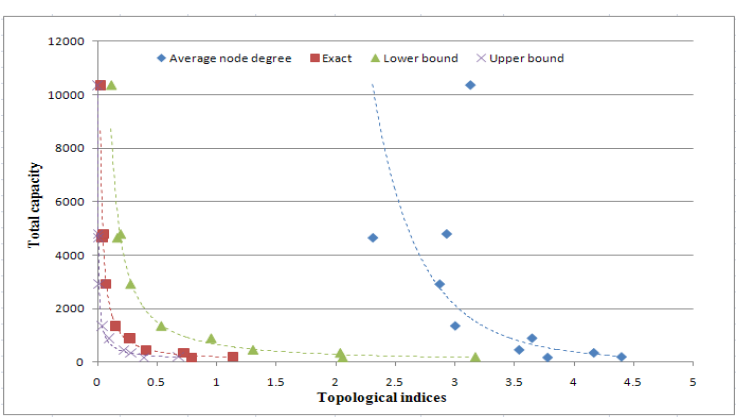

Fig.3 Total capacities vs. four topological indices

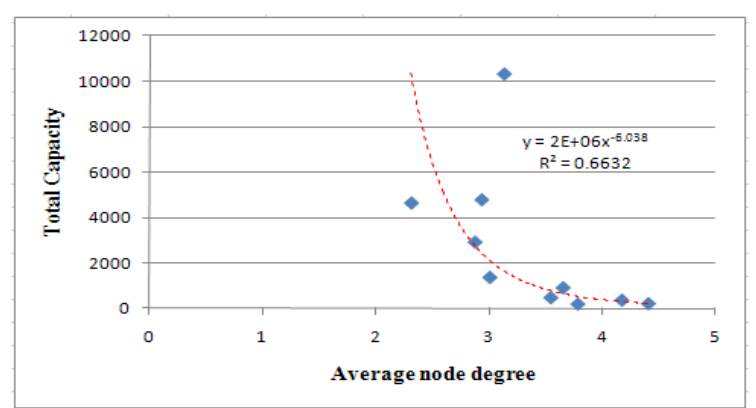

Fig.4 Total capacity vs. average nodal degree 


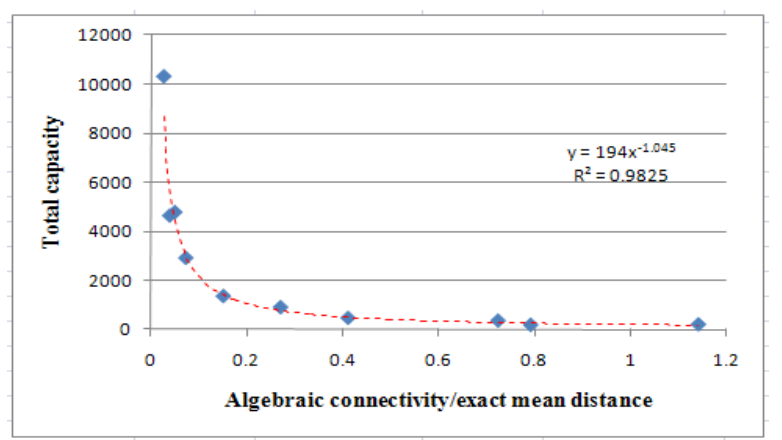

Fig.5 Total capacity vs. algebraic connectivity/exact mean distance

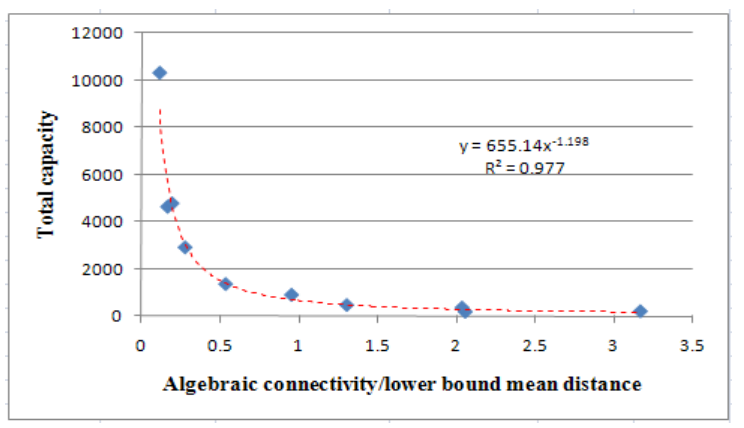

Fig.6 Total capacity vs. algebraic connectivity/lower bound mean distance

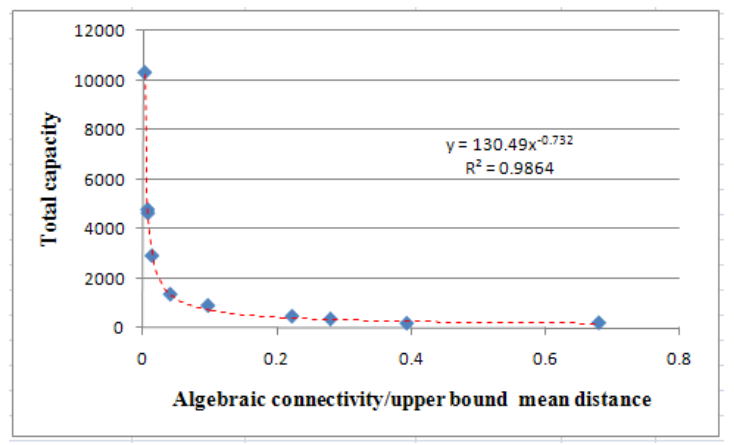

Fig.7 Total capacity vs. algebraic connectivity/upper bound mean distance

We compare four different topological indices:

- $\bar{d}$ : average nodal degree

- $\lambda_{2}(G) / \rho_{\text {exact }}(G)$ : algebraic connectivity/exact mean distance

- $\lambda_{2}(G) / \rho_{\text {loner }}(G)$ : algebraic connectivity/lower bound on mean distance

- $\lambda_{2}(G) / \rho_{\text {upper }}(G)$ : algebraic connectivity/upper bound on mean distance

To carry out our comparison, we compute for each of our ten example networks the total capacity according to the SBPP algorithm, and furthermore the values of the above topological indices. Following this, for each of our topological indices we show a scatter plot between the value of the topological index and the total capacity, one point for each example network. Since our topological indices are real-valued, we can attempt to identify a functional relationship between the topological index and the total capacity. To achieve this, we use curvefitting (specifically, we use the "trendline" function of Excel). The results are shown in Figures 2 to 7. We include in these figures the coefficient of determination, $R^{2}$. This measures the percentage of variation in the dependent variable that is explained by the fitted line. It has a value between ' 0 ' and ' 1 ', with a higher value indicating a better fit. $R^{2}=$ ' 1 'means best fitting and $R^{2}=$ ' 0 ' means worst fitting.

Firstly, we can investigate the correlation between the new topological index $\lambda_{2}(G) / \rho(G)$ and the algebraic connectivity, and also the accuracy of the lower and upper bounds on the mean distance compared with the exact one. As shown in Figure 2, we can see that, there is a growing power law correlation between the algebraic connectivity and the new proposed topological index. The values of $\rho_{\text {lower }}(G)$ and $\rho_{\text {upper }}(G)$ give the lower bound and upper bound on mean distance, thus they also give the upper and lower bounds on the new topological index: $\lambda_{2}(G) / \rho(G)$. As the algebraic connectivity increases, the curve of the $\lambda_{2}(G) / \rho_{\text {exact }}(G)$ is monotonically increasing among the curves of $\lambda_{2}(G) / \rho_{\text {lower }}(G)$ and $\lambda_{2}(G) / \rho_{\text {upper }}(G)$ with a power law trend, i.e., $y=0.5243 x^{1.3823}$. We can also find the lower and upper bounds are always falling in the following ranges of the exact mean distance based on the ten topologies:

$$
\begin{gathered}
\rho_{\text {lower }}(G) \approx\{23 \% \sim 38 \%\} \cdot \rho_{\text {exact }}(G) \\
\rho_{\text {upper }}(G) \approx\{1.68 \sim 10.24\} \cdot \rho_{\text {exact }}(G)
\end{gathered}
$$

From the equations (10-11) and Figure 2, it can be seen that the lower bound and upper bound on mean distance are all good estimation on the network mean distance. Therefore, the $\lambda_{2}(G) / \rho_{\text {lower }}(G)$ and $\lambda_{2}(G) / \rho_{\text {upper }}(G)$ have similar power law correlations to the $\lambda_{2}(G)$ as the exact one. As the algebraic connectivity increases, the accuracy of the lower and upper bounds on the mean distance become worse.

The Figure 3 gives an overview of the correlations between the total capacities and four topological indices, and we can see their relationships and numerical ranges to each other. More details of the numerical analysis for each topological index are shown in Figures 4-7. In Figure 4, we can see that there is no particularly strong correlation between the total capacity and average nodal degree index, the coefficient of determination is $R^{2}=0.6632$. For example, four network topologies have the similar average nodal degree about 3, but they have total capacity solutions varying between 1351 and 10346 units, which means the average node degree index only tell us limited information about network topology. However, for larger values of the nodal degree the correlation is better than for lower values. On the other hand, as shown in Figure 5, there is clear power law trend (with $y=194 x^{-1.045}$ ) with a good fitting of $R^{2}=0.9825$ between the total capacity associated with $\lambda_{2} / \rho_{\text {exact }}(G)$. Figure 5 shows that the total capacity obtained for different network topologies decreases with the value of the ratio $\lambda_{2}(G) / \rho_{\text {exact }}(G)$ associated with topologies. When $\lambda_{2}$ is fixed, the total capacity increases as the $\rho_{\text {exact }}(G)$ increases since as the mean distance becomes larger, and more working and protection capacity needs to be allocated. If $\rho_{\text {exact }}(G)$ is 
fixed, the total capacity decreases as the algebraic connectivity $\lambda_{2}$ increases, since a larger $\lambda_{2}$ indicates denser connectivity, and more capacity sharing can be achieved in SBPP scheme.

Additionally, it can been seen in Figures 6 and 7 that there are similar monotonic power-law trends (with $y=655.14 x^{-1.198}$ in Figure 6 and $y=130.49 x^{0.732}$ in Figure 7) with a good fitting of $R^{2}=0.977$ for $\lambda_{2}(G) / \rho_{\text {lower }}(G)$, and $R^{2}=0.9864$ for $\lambda_{2}(G) / \rho_{\text {upper }}(G)$, which are all sufficiently tight to show similar correlation as the one existing between the total capacity and the exact one. We note that for determining $\rho_{\text {lower }}(G)$, we need to know $N$ and $\lambda_{2}$ only, while for $\rho_{\text {upper }}(G)$, the parameters of $\lambda_{2}, N$ and $\Delta(G)$ are all needed. Thus, the lower bound on mean distance is easier to calculate than the upper bound, which are all derived based on the algebraic connectivity $\lambda_{2}$. On the other hand, it is a more complex procedure to calculate the exact mean distance by the shortest path finding algorithm in MATLAB and it has scalability problem as the network structure become larger. Therefore, we conclude that the algebraic connectivity metric, $\lambda_{2}$, can both used for assessing the network connectivity and for estimating the $\rho(G)$. The newly proposed topological index, i.e., $\lambda_{2}(G) / \rho(G)$ can indicate more characteristics such as size and shape of the network topology and it has a power law correlation i.e., $y=a \cdot x^{b}$ to the total capacity allocated in survivable routing design.

\section{CONCLUSIONS AND FUTURE WORK}

In this paper, we have argued that the use of average nodal degree of a network for describing its connectivity characteristic is not satisfying. We suggest using an alternative topological index: the ratio of algebraic connectivity to mean distance of the network. Our results show that for the chosen example networks it is a better quantitative descriptor of network topologies in studies of the capacity allocation problem. We have found a power law relationship between this topological index and total capacity in survivable network designed with the SBPP algorithm. This new finding can be applied into network optimization, for such as the estimation on the total capacity if the topology is given, or on the other hand, the evaluation on the topology structure if the total capacity constraints are given.

An extensive validation on our findings on more complex topologies is underway. Further studies on how to apply this new topological index into network capacity planning and optimization, e.g., one node/link addition problem are needed. In addition, how to configure the parameters such as ' $a$ ' and ' $\mathrm{b}$ ' in the power law function $y=a \cdot x^{b}$ from the characteristics of the given topology structure should be studies in future work.

\section{ACKNOWLEDGEMENT}

Thanks to Dr Allan McInnes and Fred Samandari for their valuable comments to improve this paper and their constructive suggestions for the future works. This work was partially supported by REANNZ (2008 KAREN Capability Build Fund).

\section{REFERENCES}

[1] W. D. Grover, Mesh-Based Survivable Networks: Options and Strategies for Optical, MPLS, SONET, and ATM Networking, Prentice Hall PTR, Upper Saddle River, NJ, 2004.

[2] W. D. Grover, J. Doucette, M. Clouqueur, D. Leung and D. Stamatelakis, "New options and insights for survivable transport networks," IEEE Communications Magazine, 40(1), 2002, pp. 34-41.

[3] B. Forst and W. D. Grover, "Factors affecting the efficiency of Demand-wise Shared Protection" Proc. Design of Reliable Communication Networks (DRCN 2007), pp. 1-8.

[4] B. Todd and J. Doucette, "Multi-Flow Optimization Model for Design of a Shared Backup Path Protected Network" Proc. ICC '08. IEEE International Conference on Communications, 19-23 May 2008, pp. 131 -138 .

[5] J. Doucette and W. D. Grover, " Comparison of mesh protection and restoration schemes and the dependency on graph connectivity" , Proc. 3rd Int. Workshop on the Design of Reliable Communication Networks (DRCN 2001), pp.121-128.

[6] W. D. Grover and J. Doucette, "Increasing the Efficiency of Spanrestorable Mesh Networks on Low-connectivity Graphs", Proc. 3rd Int. Workshop on the Design of Reliable Communication Networks (DRCN 2001), pp.99-106.

[7] J. Doucette, M. Clouqueur and W. D. Grover, "On the Availability and Capacity Requirements of Shared Backup Path-Protected Mesh Networks", Optical Networks Magazine, Vol. 4, No. 6, November 2003, pp. 29-44.

[8] William Liu, Harsha Sirisena, Krzysztof Pawlikowski and Allan McInnes, "Utility of Algebraic Connectivity Metric in Topology Design of Survivable Networks," in Proc. of 7th International Workshop on the Design of Reliable Communication Networks (DRCN 2009), October 2009, Washington DC, pp.131 - 138.

[9] F.R.K. Chung, Spectral Graph Theory, CBMS Regional Conference Series in Mathematics, No. 92, 1997.

[10] B. Mohar, "Some applications of Laplace eigenvalues of graphs", Graph Symmetry: Algebraic Methods and Applications, volume 497 of NATO ASI Series C, 1997, pp. 227-275.

[11] M. Fiedler, "Algebraic connectivity of graphs," Czechoslovak Mathematical, J., vol. 23, 1973, pp. 298-305.

[12] M. Fiedler, "A property of eigenvectors of nonnegative symmetric matrices and its application to graph theory," Czechoslovak Mathematical J., vol. 25, 1975, pp. 619-633.

[13] A. Bigdeli , A. Tizghadam , A.L. Garcia, "Comparison of network criticality, algebraic connectivity, and other graph metrics," Proc. 1st Annual Workshop on Simplifying Complex Network for Practitioners, July 01-01, 2009, pp.1-6.

[14] Bojan Mohar, "Eigenvalues, Diameter and Mean Distance in graphs," Graphs Combin. 7 (1991), pp. 53-64.

[15] G.Birkan, J. L. Kennington, E. Olinick, A. Ortynski and G. Spiride, "Making a Case for Using Integer Programming to Design DWDM Networks", Optical Networks Magazine, Vol. 4, No. 6, November/December 2003, pp. 107-120.

[16] Y. Liu, D. Tipper, and P. Siripongwutikorn, "Approximating Optimal Spare Capacity Allocation by Successive Survivable Routing," ACM/IEEE Transactions on Networking, Vol. 13, No. 1., pp. 198-211, Feb., 2005

[17] R. Fourer, D. M. Gay, and B. W. Kernighan, AMPL: A Modeling Language for Mathematical Programming. San Francisco, CA,1993.

[18] CPLEX User Manual v11.1, ILOG, Inc., 2008G. Iannaccone, C. Chuah, R. Mortier, S. Bhattacharyya, and C. Diot, "Analysis of link failures in an IP backbone," in Proc. of ACM Sigcomm Internet Measurement Workshop, Nov. 2002.

[19] Matlab file exchange central: http://www.mathworks.com/matlabcentral/ fileexchange/8808-all-pairs-shortest-path-graph-solver 